\title{
Action Video Game Players Do Not Differ in the Perception of Contrast-Based Motion Illusions but Experience More Vection and Less Discomfort in a Virtual Environment Compared to Non-Action Video Game Players
}

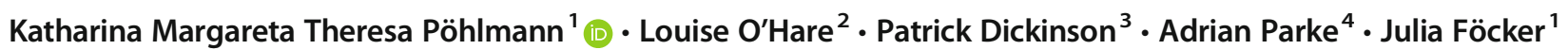

Received: 18 January 2021 / Accepted: 6 April 2021 / Published online: 20 April 2021

(C) The Author(s) 2021

\begin{abstract}
Action video game players (AVGPs) show enhanced visual perceptual functions compared to their non-video game playing peers (NVGPs). Whether AVGPs are more susceptible towards static contrast motion illusions, such as Fraser Wilcox illusions, has not been addressed so far. Based on their improved perceptual skills, AVGPs are expected to be more susceptible to the illusions and perceive more motion in them. The experience of illusory self-motion (vection) is believed to be dependent on top-down attentional processes; AVGPs should therefore experience stronger vection compared to NVGPs based on their improved attentional skills. Lastly, due to their extensive prior experience with virtual environments, AVGPs should experience less discomfort in VR compared to NVGPs. We presented rotating and expanding motion illusions in a virtual environment and asked 22 AVGPs and 21 NVGPs to indicate the strength of illusory motion, as well as the level of discomfort and vection experienced when exposed to these motion illusions. Results indicated that AVGPs and NVGPs perceived the same amount of motion when viewing these illusions. However, AVGPs perceived more vection and less discomfort compared to NVGPs, possibly due to factors such as enhanced top-down attentional control and adaptation. No differences in the perception of expanding and rotating illusions were found. Discomfort experienced by AVGPs was related to illusion strength, suggesting that contrast illusions might evoke the perceived discomfort rather than the virtual environment. Further studies are required to investigate the relationship between contrast sensitivity, migraine and the perception of illusion in AVGPs which should include illusory motion onset and duration measures.
\end{abstract}

Keywords Perception $\cdot$ Video games $\cdot$ Virtual reality $\cdot$ Vection $\cdot$ Discomfort $\cdot$ Motion illusion

\section{Introduction}

Improving perceptual skills via action video game training has been discussed in relation to a wide range of therapeutic treatments. Individuals diagnosed with amblyopia, a

Katharina Margareta Theresa Pöhlmann

KPohlmann@lincoln.ac.uk

1 School of Psychology, University of Lincoln, Lincoln, UK

2 Division of Psychology, Nottingham Trent University, Nottingham, UK

3 School of Computer Science, University of Lincoln, Lincoln, UK

4 School of Media, Culture and Society, University of the West of Scotland, Glasgow, UK developmental disorder which is characterised by reduced visual acuity in one eye, showed improvement in visual acuity and binocular vision after action video game training ( $\mathrm{Li}$ et al. 2011; Vedamurthy et al. 2015a, b). Similarly, children with dyslexia revealed increased reading skills when trained with action mini games such as Raving Rabbids (Franceschini et al. 2017).

Visual information processing abilities have shown improvements in habitual action-video game players (AVGPs) as well as after action video game training (Chopin et al. 2019; Hutchinson and Stocks 2013; Riesenhuber 2004). AVGPs outperform non-action video game players (NVGPs) in various motion perception tasks. They are better at perceiving the direction of motion in random dot kinematograms (Pavan et al. 2016), they are faster at choosing the perceived motion direction (left or right) (Green et al. 2010) and outperform 
NVGPs in motion object tracking tasks (Green and Bavelier 2006). Better performance in motion tracking tasks has been observed for habitual AVGPs and after training and is believed to be related to improvements in attentional control functions, such as the ability to filter irrelevant information while focusing on task-relevant information (Bavelier and Green 2019). Finding positive implications not only for habitual video game players but also after video game training would suggest that video game players are not predisposed to have a better motion perception which would contribute to them becoming habitual players, but rather that the game play itself causes these improvements in visual skills.

These studies mainly discussed motion perception elicited by physically moving patterns when comparing gamers with non-gamers. In the current study, however, we are interested whether AVGPs differ from NVGPs in the perception of motion illusions by showing a stronger illusion percept than NVGPs and perceiving the stationary patterns as moving more. We present these illusions in a virtual environment because we aimed to answer the question whether illusions can be perceived even if they are presented virtually and whether any prior experience with video gaming can modulate this perceptual experience. We also examined this question while participants were running the experiment on a computer desktop in order to test whether the chosen device has an impact on the experience of motion illusions and whether we observe any differences between gamers and non-gamers. As those illusions elicit motion, we were also interested in any discomfort the observer experiences via these illusions, and whether gamers differ from non-gamers in perceived discomfort. This question is of relevance, as an in depth understanding of how illusions are perceived in virtual environments provides further answers to the question about the plasticity of cognitive, perceptual and subjective experiences in a virtual environment and how much our perceptual senses are able to 'virtually' perceive illusions compared to the presentation on a computer desktop. Furthermore, we were interested in the impact of any prior video game experience as previous studies have observed increased perceptual skills after video game training which might have an impact on how motion illusions are perceived as well as how this impacts discomfort. As the current experiments provide an in depth understanding about perceptual experiences including perception of motion illusions and discomfort in VR related to prior video game experiences, it might provide some important insights about creating VR applications to reduce discomfort.

The illusions used in this study are so-called optimised Fraser Wilcox illusions (Fraser and Wilcox 1979). This type of illusion consists of repeated luminance gradients (black $\rightarrow$ dark grey $\rightarrow$ light grey $\rightarrow$ white; see Figs. 1 and 2) with illusory motion being perceived from dark to light shading and appearing in a constant direction guided by these patterns (Fraser and Wilcox 1979; Kitaoka 2006; Kitaoka and Ashida
2003; Shapiro and Todorovic 2016). The motion is perceived due to differences in contrast and luminance which are associated with differences in neural processing speed which can lead to motion detectors in the brain producing sequential neuronal responses similar to those of physically moving stimuli (Backus and Oruç 2005; Conway et al. 2005; Otero-Millan et al. 2012). These types of illusions are strongest when presented in the periphery and are often referred to as peripheral drift illusion (Faubert and Herbert 1999; Fraser and Wilcox 1979; Kitaoka and Ashida 2003; Naor-Raz and Sekuler 2000).

Eye movements have also been related to the perception of the motion illusions. It was believed that the illusion involved retinal shifts caused by small involuntary eye movements (microsaccades) that observers make while trying to maintain fixation (Murakami et al. 2006; Otero-Millan et al. 2012; Seno et al. 2013), suggesting that individuals with greater fixation instability are also more likely to experience a stronger perception of the illusion (Murakami et al. 2006). However, an eye tracking study conducted by Hermens and Zanker (2012) found that microsaccade patterns were unaffected by the strength of the Riley's Fall illusion, with their data suggesting that rather than microsaccades, slow oculomotor drifts might be contributing towards these contrast illusions (Hermens and Zanker 2012). The Riley's Fall illusion is characterised by black and white undulating lines that can induce a strong perception of 'shimmering' motion, making this illusion contrast dependent. This contrast dependence is similar to the illusions used in this study. Therefore, rather than eye movements, contrast sensitivity of the viewer might explain how the illusion occurs and could predict individual differences in perception of the illusory motion. Based on the improved perceptual skills and discrimination reported in video game players (Bejjanki et al. 2014; Li et al. 2009, Li et al. 2010; see Chopin et al. 2019 for a review) and even after action video game training (Bejjanki et al. 2014; Li et al. 2009, 2010), we hypothesise that AVGPs will perceive the illusions presented in the current study (Fig. 2) as moving more compared to NVGPs.

Motion stimuli, including illusions of motion, can induce a sensation of illusory self-motion in a stationary observer, known as vection (Dichgans and Brandt 1978; Fischer and Kornmüller 1930). Experiencing vection while exploring a virtual environment can enhance the feeling of presence in the user by eliciting a more realistic sensation of self-motion through the virtual environment and thereby improving the user's experience of VR (Riecke 2011). The visual stimuli inducing the illusory self-motion can be explicit (physically moving) or implicit (illusory) in nature. Traditionally, physically moving visual stimuli such as optic flow patterns (random dot stereograms) have been used to study vection, but research suggests that in order to experience vection the visual stimulus does not necessarily require explicit motion (Seno et al. 2013). According to that theory, the perceived motion, 
Fig. 1 Example of an a expanding optimised Fraser Wilcox illusion and the $\mathbf{b}$ corresponding stimulus used in this study (presented for 90s)
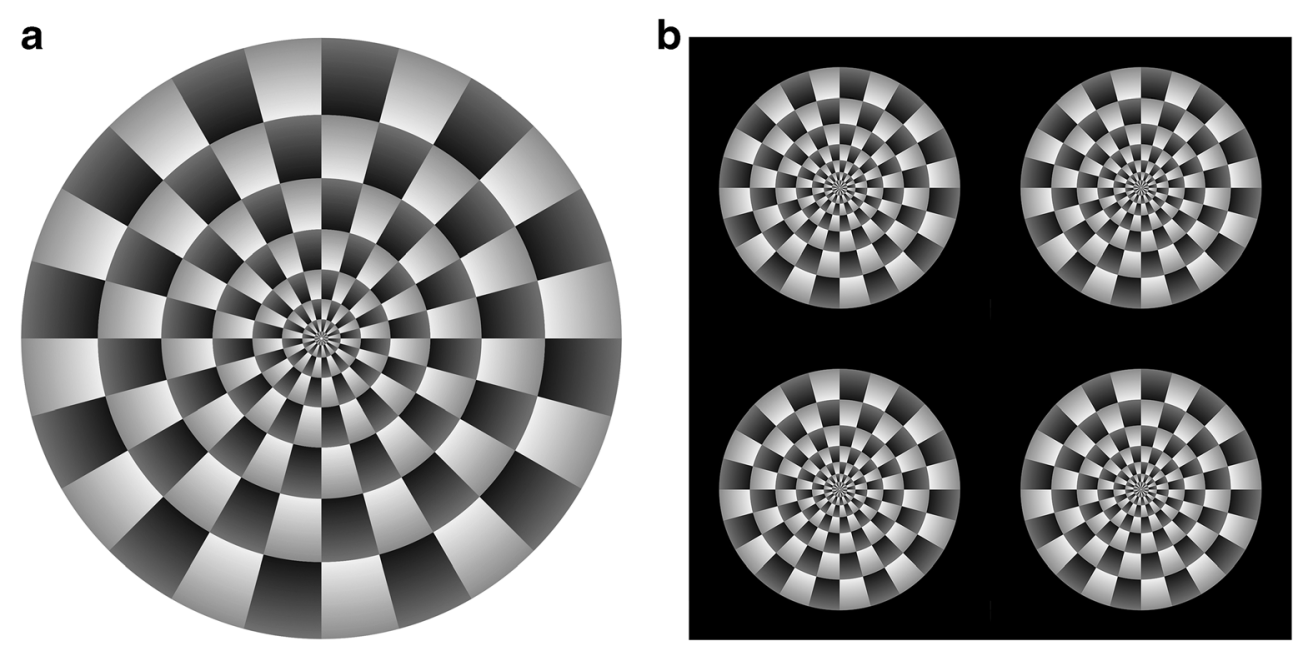

rather than the physical stimulus motion, causes vection (Nakamura 2013; Seno et al. 2012; Seno et al. 2013; Seno and Sato 2011). Vection can be elicited by motion aftereffects (Seno et al. 2010) as well as by illusory motion (Seno et al. 2013). In their study, a colourful expanding optimised Fraser Wilcox illusion was able to elicit the illusion of self-motion in the observers. Contradictory findings showed that a rotating motion illusion did not induce self-motion in observers (Rosenblatt and Crane 2015). The motion illusions used in their experiment consisted of static, repeated asymmetric patterns mapped onto a torus, giving the illusion of the inside of the torus either rotating clockwise or counterclockwise. Even though both studies used optimised Fraser Wilcox illusions as stimuli, they differed in some aspects; Seno and colleagues illusions were simple expanding illusions eliciting motion-in depth whereas the illusions used by Rosenblatt and Crane's gave the illusion of the observer being in the inside of a torus which rotated around them either to the left or the right. It is possible that this type of motion is less suitable to induce selfmotion in an observer. These contradictory results motivated the choice of both rotating and expanding motion illusions in this study to ensure that illusory motion either in the lateral direction or moving in depth could elicit vection.

Different stimulus properties can affect the experience and strength of vection, such as the size (e.g. Berthoz et al. 1975; Brandt et al. 1973; Nakamura 2006; Telford and Frost 1993) and speed (e.g. Allison et al. 1999; Nakamura and Shimojo 1999; Seya et al. 2014, 2015) of presented stimuli. As vection increases with increasing speed of the visual stimulus, we expect stronger motion illusions, images that are perceived as moving more, to also elicit stronger vection. AVGPs are expected to perceive the illusions as moving more; therefore, we also expected them to experience more vection while looking at them compared to NVGPs.

More recent research suggests that vection is not only a bottom-up process that is influenced by stimulus properties but is also affected by cognitive factors (Riecke et al. 2006). For example, participants' experience of vection could be affected by biased instructions of the researcher prior to the experiment either priming them for self-motion or object motion (Palmisano and Chan 2004), suggesting some form of top-down processing involved in the experience of vection. a
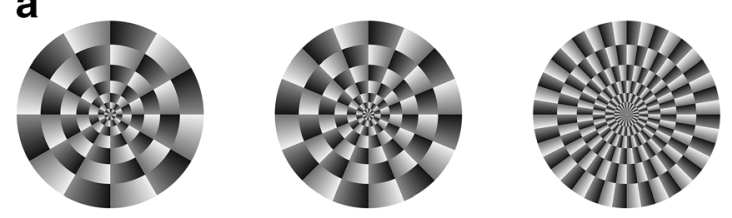

b

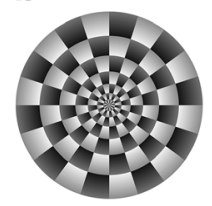

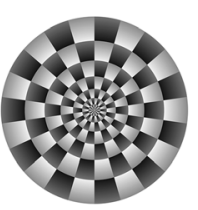

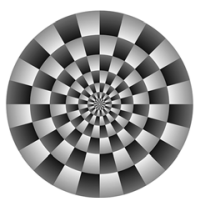

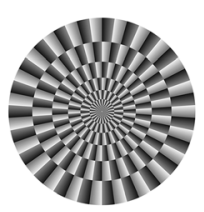
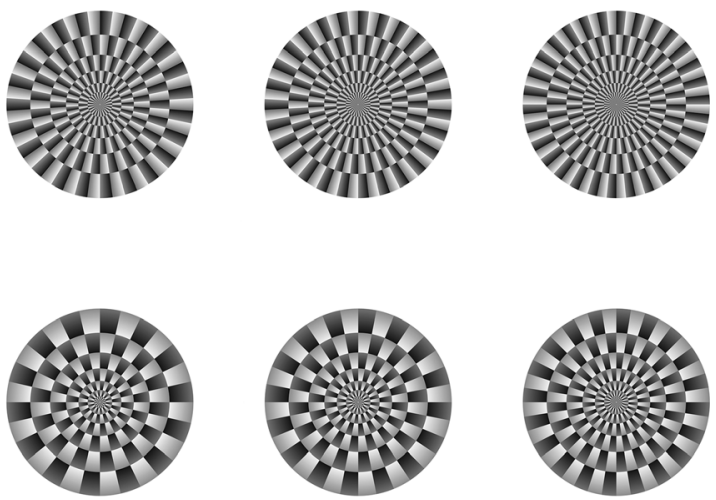

Fig. 2 The 12 stimuli used in this study. a Displaying the rotating motion illusions and $\mathbf{b}$ displaying the expanding motion illusion 
Auditory vection studies have shown that moving sounds associated with stationary objects (e.g. church bell) can induce a stronger sensation of vection compared to sounds associated with moving objects (e.g. car; Riecke et al. 2005). Studies investigating the effect of cognitive/attentional load on the experience of vection found that performing tasks requiring high attentional load resulted in a weaker experience of vection compared to control conditions even if the moving stimulus eliciting the vection was identical for both tasks (Seno et al. 2011).

Playing action video games has been related to improvements in attentional control functions as well as cognitive control (Anguera et al. 2013; Bavelier et al. 2012; Bavelier and Green 2019; Bediou et al. 2018; Boot et al. 2008; Chisholm et al. 2010; Chisholm and Kingstone 2012; Föcker et al. 2018, 2019) which would suggest that if vection is a cognitive top-down process, AVGPs should perceive a stronger percept of vection. To our knowledge, no study so far has looked at the relationship between video gaming behaviour and vection induced by perceived motion directly. Harvey and colleagues (2015) conducted a study investigating the differences in experienced vection between video game players and NVGPs for contracting and expanding optic flow patterns finding that more experienced video game players felt a stronger sensation of backwards illusory self-motion for contracting optic flow patterns compared to less experienced video game players, suggesting that differences in the experience of vection between gamer groups could be affected by the direction of the presented motion stimulus. Based on these findings, illusions eliciting motion in two directions (rotating and expanding) were chosen for this study to investigate if differences in vection found between gamer groups are related to the direction of perceived motion.

Discomfort experienced in virtual environments has been linked to vection and is particularly prevalent in HMDs (Keshavarz et al. 2015). The term discomfort used in this paper describes adverse physiological effects experienced in VR, such as motion sickness-like symptoms (e.g. nausea, disorientation, dizziness) and visual stress symptoms (e.g. headache, eye strain, difficulty focusing). Traditionally, vection was believed to cause or at least precede discomfort (Hettinger et al. 1990). Multiple studies have found a positive relationship between vection and discomfort (e.g. Diels et al. 2007; Moss and Muth 2011; Palmisano et al. 2007) whereas other studies have found contradictory results, suggesting that vection does not necessarily cause discomfort or is even related to it at all (e.g. Bonato et al. 2008; Keshavarz et al. 2015; Palmisano et al. 2017; Webb and Griffin 2003). Individual differences might help explain this complex relationship between discomfort and vection experienced in virtual environments. In the current study, the relationship is investigated based on participants' prior experience with video games.
Two main causes of discomfort in VR are the 'accommodation-vergence conflict' (e.g. Hoffman et al. 2008; Kramida 2015), which is a conflict within the visual focusing system, and the conflict between the visual and the vestibular system (e.g. Akiduki et al. 2003). VR sickness caused by the latter is often accompanied by postural instability and 'sway' (Akiduki et al. 2003; Arcioni et al. 2019; Smart et al. 2002).

Studying these two causes separately is important in understanding how to mitigate their effects; however, finding stimuli which enable this is not as straightforward. In this study, we take a novel approach by using optimised Fraser Wilcox illusions to study sickness caused by perceived motion. These illusions are 2D stationary images that, due to their patterns, are perceived as moving, and may therefore be used to stimulate feelings of discomfort associated with motion. However, they do not actually move through the virtual environment coordinate system, and therefore minimise discomfort caused by constant change in discrepancy between accommodation and vergence.

Discomfort or motion sickness in the real world as well as in virtual environments has been shown to decrease with repeated exposure. Repeatedly exposing an individual to the same sickness-inducing environment (real or virtual) has been shown to reduce adverse symptoms, such as in sea travel (Li et al. 2012), driving or flight simulators (Domeyer et al. 2013; Heutink et al. 2019; Kennedy et al. 2000) or virtual environments presented using HMDs (Hill and Howarth 2000; Howarth and Hodder 2008; Regan 1995). Habituation has been argued to be one of the most effective techniques to minimise discomfort experienced in virtual environments (Keshavarz 2016); however, adaptation can be time consuming and requires motivation in the user. In this study, we are interested if adaptation effects are dependent on repeated exposure to the exact same virtual environment and visual stimulus or if similar adaptation effects can occur when prior experience with virtual environments was gained using different display and set up types. We were interested whether AVGPs that played video games using computers or gaming consoles build up adaptation effects that would lead to them experiencing less discomfort in a virtual environment presented using HMD compared to NVGPs. In line with this notion, previous research has found that gamers experience less motion sickness when viewing videos on large projector screens or when performing a simulated driving task (Keshavarz 2016; Ujike et al. 2008).

In the current study, we presented rotating and expanding Fraser Wilcox illusions in VR using HMDs and we asked our participants to rate the strength of the illusion and the degree of vection and discomfort they experienced. Additionally, participants indicated the onset and duration of vection by button press. AVGPs are more frequently exposed to digital environments and thus can be expected to experience less discomfort in those virtual environments. According to the principles of 
habituation, we argue that action video game players might adapt more to virtual environments and therefore experience less discomfort when presented with visual stimuli in a $3 \mathrm{D}$ virtual environment.

\section{Method}

\section{Participants}

Forty-three participants ranging in age from 19 to 39 years $(\mathrm{M}=21.00$ years, $\mathrm{SD}=4.17)$ with normal or corrected to normal vision took part in this study. Twenty-four participants identified as female, and 19 as male. Twenty-two participants were classified as AVGPs (15 males, 7 females, mean age $=$ 20.27 years) and 21 as NVGPs (4 males, 17 females, mean age $=21.76$ years). Nineteen participants had previous experience with VR whereas the other 24 had never used VR before. Out of the 19 participants that had used VR before, seven participants classified as NVGPs and 12 as AVGPs, with only one of the participants having used it on a regular basis and others having used it once or twice before. Written informed consent was obtained from all participants prior to participating in the experiment and the study was approved by the University of Lincoln's Ethics Committee. Additionally, participants were informed that they could withdraw from the study at any point and that data would be analysed anonymously. Individuals suffering from photosensitive epilepsy as well as pregnant individuals were excluded from the study.

\section{Apparatus}

A PC with Intel i7-7700 core processor, 16GB RAM and an NVidia graphics card, running 64-bit Windows, 10 was used to control the headset. A Valve Index headset was used to present the stimuli with a display resolution of $1440 \times 1600$ pixels per eye, refresh rate of $120 \mathrm{~Hz}$ and yields a FOV of up to $130^{\circ}$ depending on observers' settings. Stimuli were presented using 64-bit Unity 2018.2 (Unity Technologies, San Francisco, USA), using the Steam platform.

\section{Stimuli}

The stimuli used in this study consisted of four circular optimised Fraser Wilcox illusions, each being made up by eight rings containing patterns with gradient luminance profiles (black to dark grey and white to light grey), see Fig. 1. The 12 illusions (6 rotating and 6 expanding, see Fig. 2) used were chosen based on pilot work trying to create rotating and expanding motion illusions that were similar in illusion strength. Illusion strength indicates how much motion is perceived in the illusion. The illusions were presented in the centre of the visual field covering around $23^{\circ}$ of the FOV at a distance of 10 units (unity measure of size and distance), see Fig. 3. Distance and size of stimuli were chosen (based on pilot work) to elicit the strongest motion perception in the patterns.

\section{Subjective Measures}

\section{Gamer Group}

Participants were categorised as AVGPs and NVGPs according to the criteria of the Video Game Playing Questionnaire created by the Bavelier lab (Bavelier 2019). Participants were classified as AVGPs when they currently played at least $5 \mathrm{~h}$ of action video games per week and no more than $3 \mathrm{~h}$ of all other game genres. NVGPs were individuals that, including all game genres, spent less than $3 \mathrm{~h}$ a week playing video games throughout the last year.

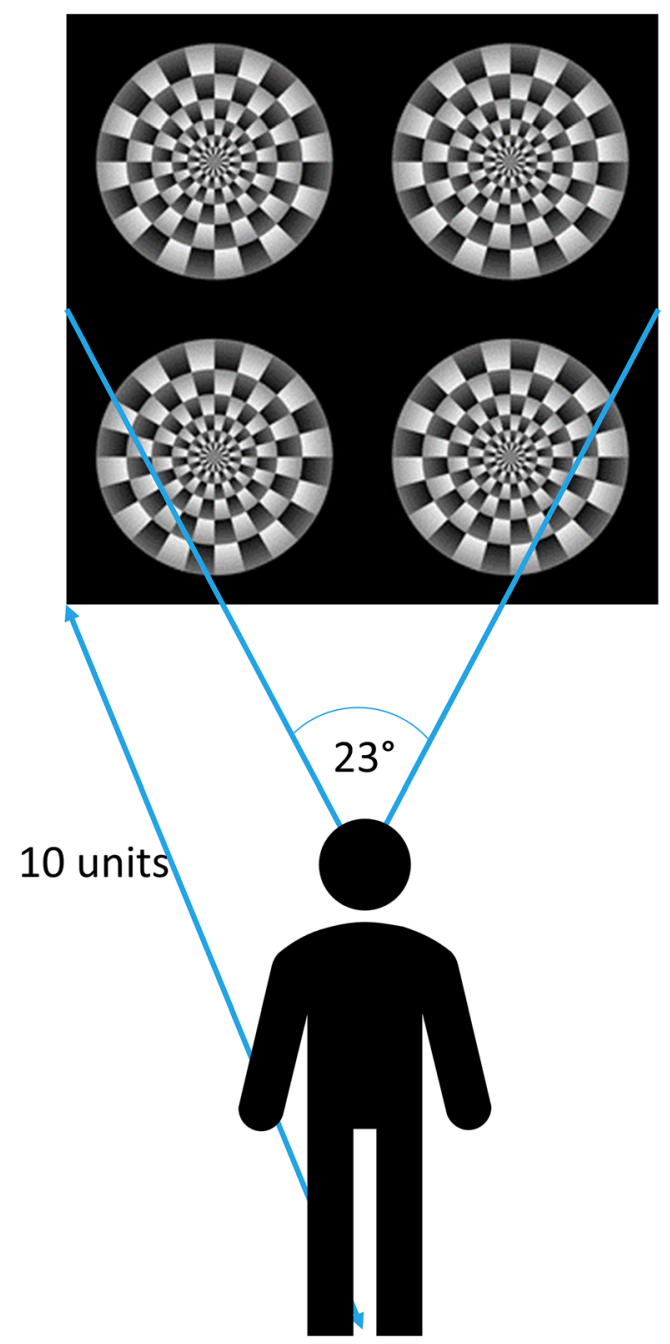

Fig. 3 The experimental set up in the virtual environment. The Stimuli were presented at a distance of 10 unity units covering around $23^{\circ}$ of the participants' field of view. The surrounding in the experiment was completely black 


\section{Illusion Strength}

For each stimulus, participants were asked to verbally rate how much motion they perceive in the illusions from ' 0 ', representing no motion at all to ' 10 '. This brief verbal assessment was performed in order to obtain illusion strength ratings for each distinct stimulus and to be able to compare illusion strength ratings for rotating and expanding motion illusions.

\section{Vection}

Vection magnitude, onset and duration were recorded as measures of vection in this study. Participants verbally rated the vector magnitude for each stimulus on an 11-point Likert scale with 0 meaning no vection at all and 10 meaning strong vection after the presentation of each stimulus. This measure reflects the magnitude of the experienced vection. In addition, vection onset and duration were recorded by button press.

\section{Discomfort}

For each stimulus, participants were asked to verbally rate their level of general discomfort, headache, blurred vision, dizziness and eye strain on an 11-point Likert scale. This brief verbal assessment was performed in order to obtain discomfort ratings for each distinct stimulus. These five ratings were aggregated in one overall discomfort measure.

\section{Procedure}

Participants filled out the gamer questionnaire online and if they qualified as either an AVGP or NVGP they were invited to take part in the lab-based VR study.

In the lab, participants were provided with an information sheet outlining the objectives and the procedure of the study. They were then asked to complete a consent form. Following, they started the VR part of the study, with participants standing in the centre of the experimental area wearing the headset and holding the controllers. To adjust to the virtual environment and to get used to controllers and headset, participants were allowed to play a VR-game prior to completing the experimental task, which lasted around 5-10 min. They played the Spiderman: Homecoming-VR Experience (Create VR, Sony Pictures VR, 2017). All participants both NVGPs and AVGPs played the video game prior to the experiment; therefore, any adverse effects caused by the game should be found for both groups. However, none of the participants reported any symptoms of discomfort and consequently withdrew at this point of the study, hence were happy to progress on to the experiment.

Participants were shown examples of the type of motion illusions they were going to view in the VR (see Fig. 2). They were instructed that it is a free viewing condition and to perceive most movement in the illusion they should not focus on any specific point on the image but rather 'wander with their eyes over the image'. They were also informed that the motion illusion will most likely be strongest in their periphery. To ensure that the illusory motion perceived when viewing the stimuli was not confused with illusory self-motion experienced in the virtual environment, the concept of vection was also explained to the participants giving the 'train example' as a real-life instance of illusory self-motion.

The environment within the experiment consisted of a completely black surrounding, which prevented any distraction from task-irrelevant visual input. The experiment consisted of two parts: a training and the experimental trial. Both the training and experimental trial started with the presentation of text informing the participant that pressing the ' $\mathrm{A}$ ' button will allow them to move on. At this point, participants were instructed to try out the different settings of the headset to get the text as focused as possible. When participants were satisfied with the focus of the headset, they could initiate the training by pressing the ' $\mathrm{A}$ ' button on the controller. In the training trial, the 12 illusions were presented one time each one after the other in random order for $5 \mathrm{~s}$ after which they disappeared, and the text screen came up again prompting the participant to press the ' $A$ ' button to move on to the next illusion. In this short training, participants were able to practise pressing the trigger button when they experienced vection in the trials, got familiar with the type of stimuli that were going to be presented in the experimental trial and they familiarised themselves with the procedures. In the experimental trial, the 12 illusions were presented in random order for $90 \mathrm{~s}$ after which they disappeared, and the text screen came up again prompting the participant to press the ' $\mathrm{A}$ ' button to move on to the next illusion. Each illusion was presented once, resulting in 12 trials in total (6 expanding and 6 rotating illusions). Participants were instructed to indicate when they started experiencing vection (vection onset) by pressing a button and to hold it in for as long as the experience of vection lasted (vection duration). They were able to press the button multiple times per trial. After each stimulus presentation, participants verbally rated the strength of the illusion as well as their experience of vection magnitude and discomfort on an 11-point Likert scale. After rating each stimulus, the researcher prompted the participant to initiate the next trial.

\section{Analysis}

Statistical analysis was conducted using R 1.2.5019 (R Core Team, 2019) using the $\mathrm{glm}$ and lmer function of the lme4 package (Bates et al. 2014) to perform generalised linear and linear mixed effect analyses on the effect gamer type on illusion strength, vection magnitude and discomfort ratings as well as on vection onset and duration times. 
Illusion strength, vection magnitude and discomfort ratings were predicted using generalised linear models with gamma distribution as data were positively skewed. Prior analysis using a generalised mixed effect model including participant variation as a random effect showed low participant-level variance resulting in the random effect being omitted from the final analysis.

Linear mixed effect models were used to analyse vection onset and duration, as these data consist of time-series for each participant and for each stimulus, which are not independent observations. Linear mixed effect models have advantages in their ability to model non-linear, individual characteristics (Krueger and Tian 2004). Additionally, they allow for multiple observations from the same participant and deal with not normally distributed and skewed data. Therefore, these models were preferred over traditional ANOVAs.

For the models, $p$ values of overall effects were determined using conditional $F$ tests with Satterthwaite's approximation to degrees of freedom (Satterthwaite 1946) using a Type III ANOVA, as implemented in the 'anova' function from the lmerTest package (Kuznetsova et al. 2017). Estimated marginal means and standard errors were calculated using the emmeans function of the emmeans package (Lenth et al. 2018). Following the examples of Winter (2013), models were created for fixed effects (predictor variables) that showed a significant effect on the outcome variable. These models were compared to the null models missing the variable of interest using a likelihood ratio test, in order to obtain a difference in Bayes Information Criterion $(\triangle \mathrm{BIC})$, to estimate the strength of the evidence for a particular effect. $\triangle \mathrm{BIC}$ is a criterion for model selection as it calculates a model's likelihood and can be seen as a way to estimate something comparable to the effect size of a predictor (Schwarz 1978). The model with the lowest $\triangle \mathrm{BIC}$ value is determined as the best fit model. Differences lower than 2 between two models are very weak and can be ignored or have to be interpreted with care. A negative difference in $\triangle \mathrm{BIC}$ indicates evidence in support of the null model, rather than the alternative model (Berchtold 2010; Kass and Wasserman 1995). $\triangle \mathrm{BIC}$ values reported here represent the difference between the full model's and the null model's $\triangle \mathrm{BIC}$ values.

For all the models, Satterthwaite's approximation was used to adjust the degrees of freedom for violations of sphericity (Luke 2017; Satterthwaite 1946). To investigate the relationship of illusion strength, vection measures and discomfort, a Spearman correlation was conducted using the 'rcorr' function within the Hmisc package (Harrell and Harrell 2019).

Figures were produced using the emmip function within the emmeans package (Lenth et al. 2018) and the plot function within the graphics package (Team 2019) as well as the 'visreg' function within the visreg package (Breheny and Burchett 2017).

\section{Results}

\section{Illusion Strength}

Illusion strength was predicted using a generalised linear model including Gamer type (AVGP vs. NVGP), Illusion type (rotating vs. expanding) and their interaction as fixed effects. The function of the model looks as follows:

Model $=\operatorname{glm}\left(\right.$ Illusion Strength $\sim$ Gamer Type ${ }^{*}$ Illusion Type)

No significant effect of gamer type $(F(1,514)=0.71, p=$ $0.399)$, illusion type $(F(1,513)=2.02, p=0.156)$ or their interaction $(F(1,512)=0.38, p=0.540)$ on illusion strength ratings was found, see Fig. 4 . The same result pattern was observed in a larger sample in a follow-up study using the computer desktop; the results of this study can be found in the supplementary material.

\section{Vection}

Vection magnitude was predicted using a generalised linear model including Gamer type (AVGP vs. NVGP, Illusion type (rotating vs. expanding) and their interaction as fixed effects. The function of the model looks as follows:

Model $=\operatorname{glm}\left(\right.$ Vection Magnitude $\sim$ Gamer Type ${ }^{*}$ Illusion Type $)$

Vection onset and duration were predicted using a linear mixed effect model including Gamer type (AVGP vs. NVGP), Illusion type (rotating vs. expanding) and their interaction as fixed effects and participant as random effect. Residual plots for the models were inspected and revealed no violation of linearity as well as homoscedasticity in vection onset/ duration times. The function of the model looks as follows

Model $=$ lmer $($ Vection Onset/Duration $\sim$ Gamer Type*Illusion Type +

A significant effect of gamer type on vection magnitude ratings was found, $F(1,514)=4.46, p=0.035, \Delta \mathrm{BIC}=$ -12.29 . AVGPs $(M=2.52,0.18 \pm \mathrm{SE}), 95 \%$ CI $[2.19,2.99]$ experienced more vection compared to NVGPs $(M=2.05$, $0.15 \pm \mathrm{SE}),[1.77,2.43]$. No significant effect of Illusion type $(F(1,513)=0.01, p=0.933)$ or their interaction $(F(1,512)=$ $0.23, p=0.628$ ) on vection magnitude ratings was found, see Fig. 5.

\section{Vection Onset}

No significant effect of gamer type $(F(1,31.42)=0.15, p=$ $0.704)$, Illusion type $(F(1,280.04)=0.25, p=0.615)$ or their interaction $(F(1,280.04)=0.12, p=0.727)$ on vection onset times was found. 
Fig. 4 Estimated 95 confidence intervals for illusion strength ratings for AVGPs and NVGPs. The dark dots representing the model points, the blue and green bars representing the $95 \%$ confidence intervals and the arrows are for comparison among the groups if an arrow from one mean overlaps an arrow from another group the difference between them is not 'significant', $(p>0.05)$

Fig. 5 Estimated 95 confidence intervals for vection magnitude ratings for AVGPs and NVGPs. The dark dots representing the model points, the blue and green bars representing the $95 \%$ confidence intervals and the arrows are for comparison among the groups if an arrow from one mean overlaps an arrow from another group the difference between them is not 'significant', $(p>0.05)$

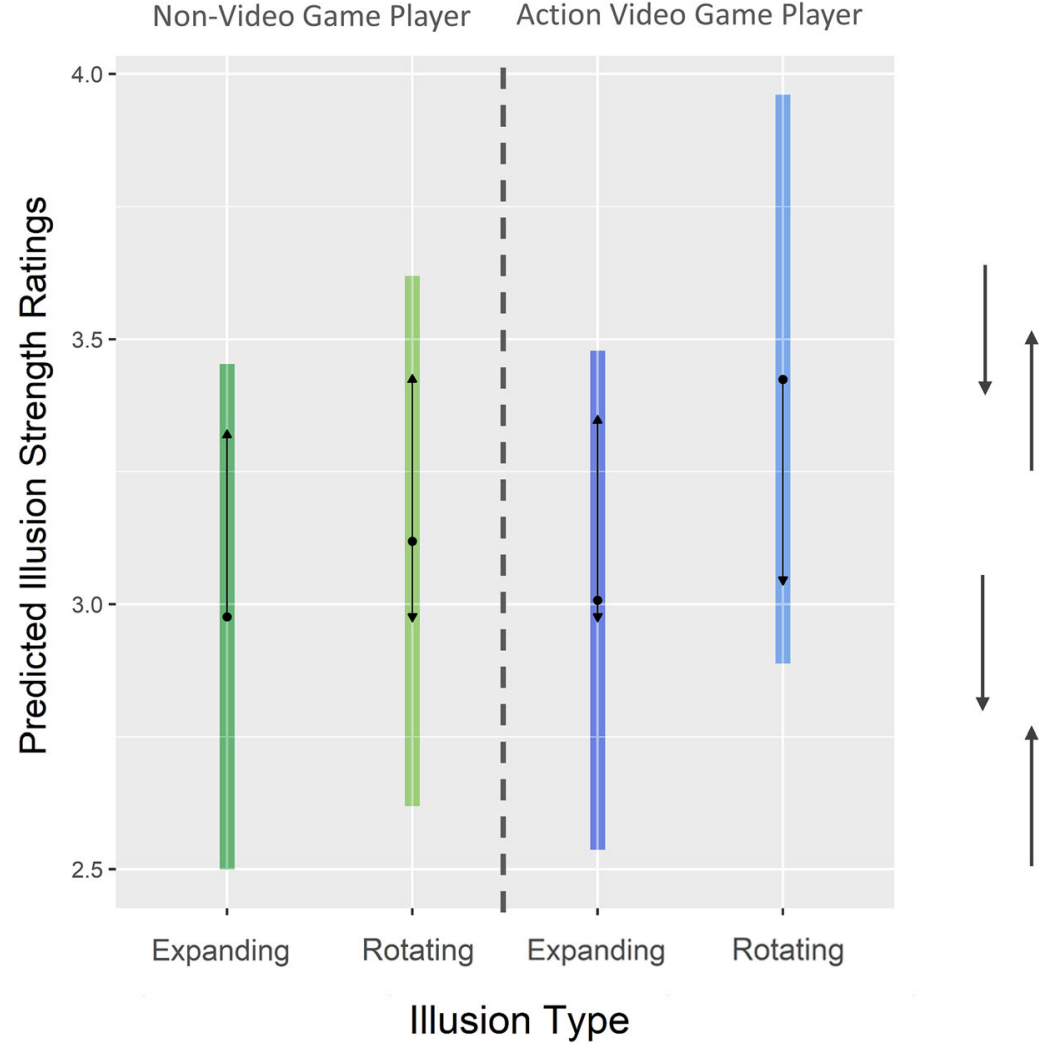

Overlapping arrows indicate no significant difference

Non-Overlapping arrows indicate significant difference

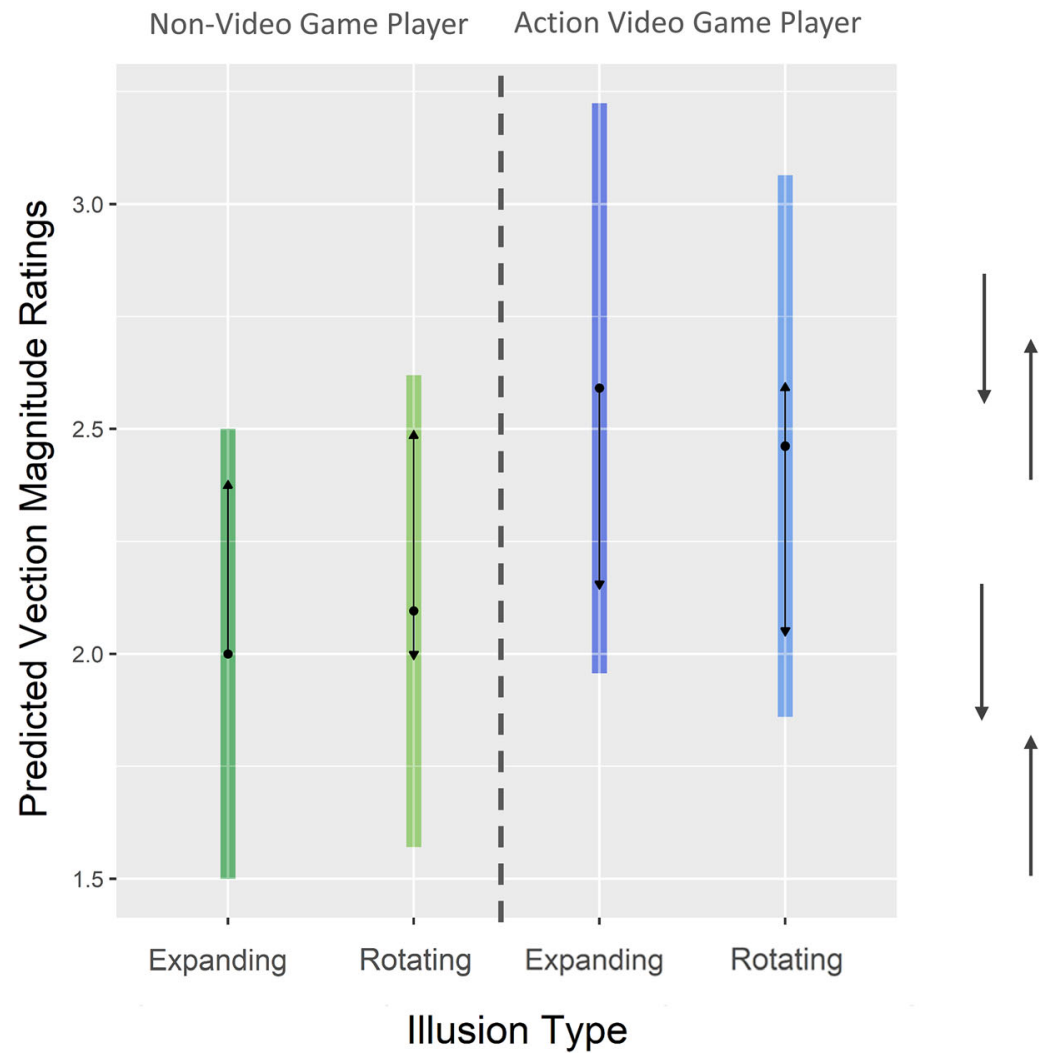

Overlapping arrows indicate no significant difference

Non-Overlapping arrows indicate significant difference 


\section{Vection Duration}

No significant effect of gamer type $(F(1,37.13)=1.33, p=$ $0.256)$, Illusion type $(F(1,281.79)=0.01, p=0.912)$ or their interaction $(F(1,281.79)=1.34, p=0.248)$ on vection duration was found.

\section{Discomfort}

Discomfort was predicted using a generalised linear model including Gamer type (AVGP vs. NVGP), Illusion type (rotating vs. expanding) and their interaction as fixed effects. The function of the model looks as follows:

Model $=\operatorname{glm}\left(\right.$ Discomfort $\sim$ Gamer Type ${ }^{*}$ Illusion Type $)$

A significant effect of gamer type on discomfort ratings was found, $F(1,514)=12.26, p<0.001, \Delta \mathrm{BIC}=-11.74$. AVGPs $(M=0.86,0.07 \pm \mathrm{SE}),[0.73,1.03]$ experienced less discomfort compared to NVGPs $(M=1.26,0.10 \pm \mathrm{SE}),[1.07$, 1.53]. No significant effect of illusion type $(F(1,513)=0.55$, $p=0.458)$ or their interaction $(F(1,512)=0.02, p=0.878)$ on discomfort ratings was found, see Fig. 6 . The five discomfort ratings (general discomfort, headache, blurred vision, dizziness and eye strain) showed a similar pattern in the analysis; therefore, the aggregated discomfort is presented here. The analysis for the five separate discomfort ratings can be found in the supplementary information.

\section{Correlation for Illusion Strength, Vection and Discomfort}

A Spearman correlation was conducted to investigate the relationship between perceived illusion strength, vection measures and experienced discomfort for rotating and expanding illusions for NVGPs and AVGPs, respectively. Spearman's rho and significance values can be found in Tables 1 and 2 . For AVGPs, a strong positive correlation was found for illusion strength and vection magnitude for both rotating $\left(\mathrm{r}_{\mathrm{s}}=\right.$ 0.66, $p<0.001, N=22)$ and expanding illusions $\left(\mathrm{r}_{\mathrm{s}}=0.71\right.$, $p<0.001, N=22$ ) and a moderate positive relation between illusion strength and discomfort for both rotating $\left(\mathrm{r}_{\mathrm{s}}=0.52\right.$, $p=0.012, N=22)$ and expanding illusions $\left(\mathrm{r}_{\mathrm{s}}=0.43, p=\right.$ $0.047, N=22$ ). For rotating illusions, a moderate positive correlation was found for vection duration and magnitude measures $\left(\mathrm{r}_{\mathrm{s}}=0.48, p=.031, N=20\right)$ and a moderate negative correlation for vection onset and duration measures $\left(\mathrm{r}_{\mathrm{s}}=\right.$ $-.52, p=0.018, N=20$ ). For expanding illusions, a moderate positive relationship between vection magnitude and discomfort was found $\left(\mathrm{r}_{\mathrm{s}}=0.44, p=0.041, N=22\right)$. For NVGPs, however, illusions strength was only related to vection magnitude $\left(\mathrm{r}_{\mathrm{s}}=0.51, p=0.018, N=21\right)$ and duration $\left(\mathrm{r}_{\mathrm{s}}=0.64\right.$, $p=0.006, N=21$ ) for expanding illusions. Additionally, a
Fig. 6 Estimated 95 confidence intervals for discomfort ratings for AVGPs and NVGPs. The dark dots representing the model points, the blue and green bars representing the $95 \%$ confidence intervals and the arrows are for comparison among the groups if an arrow from one mean overlaps an arrow from another group the difference between them is not 'significant', $(p>0.05)$

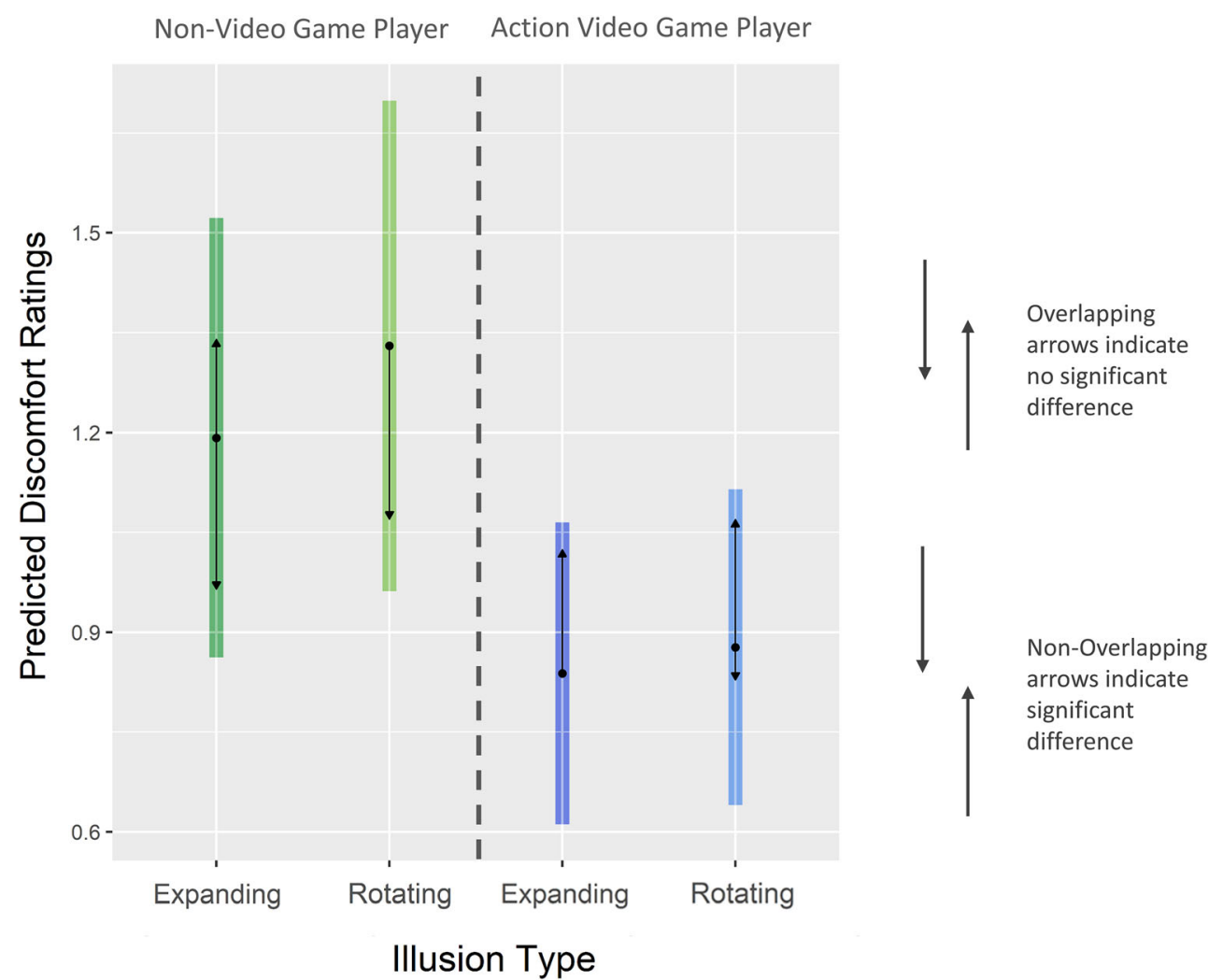


Table 1 Spearmen correlation of illusion strength ratings, vection measures (magnitude, onset, duration) and discomfort ratings for expanding and rotating illusions for NVGPs

\begin{tabular}{|c|c|c|c|c|c|c|c|c|}
\hline & \multicolumn{4}{|l|}{ Rotating } & \multicolumn{4}{|c|}{ Expanding } \\
\hline & $\begin{array}{l}\text { Illusion } \\
\text { strength }\end{array}$ & $\begin{array}{l}\text { Vection } \\
\text { magnitude }\end{array}$ & $\begin{array}{l}\text { Vection } \\
\text { onset }\end{array}$ & $\begin{array}{l}\text { Vection } \\
\text { duration }\end{array}$ & $\begin{array}{l}\text { Illusion } \\
\text { strength }\end{array}$ & $\begin{array}{l}\text { Vection } \\
\text { magnitude }\end{array}$ & $\begin{array}{l}\text { Vection } \\
\text { onset }\end{array}$ & $\begin{array}{l}\text { Vection } \\
\text { duration }\end{array}$ \\
\hline $\begin{array}{l}\text { Vection } \\
\text { magnitude }\end{array}$ & 0.34 & - & & & $0.51^{*}$ & - & & \\
\hline Vection onset & -0.33 & $-0.67 * *$ & - & & -0.31 & -0.40 & - & \\
\hline Vection duration & 0.36 & $0.65 * *$ & -0.44 & - & $0.64 * *$ & $0.83 * * *$ & -0.29 & - \\
\hline Discomfort & 0.40 & 0.41 & -0.13 & -0.28 & 0.21 & 0.24 & 0.26 & 0.06 \\
\hline
\end{tabular}

${ }^{*} p<0.05 ; * * p<0.01 ; * * * p<0.001, N=21, N_{\text {Onset Rotating }}=16, N_{\text {Onset Expanding }}=17, N_{\text {Duration Rotating }}=16, N_{\text {Duration Expanding }}=17$

strong positive correlation between vection magnitude and vection duration was found for expanding $\left(\mathrm{r}_{\mathrm{s}}=0.83, p<0.001\right.$, $N=16)$ and rotating illusions $\left(\mathrm{r}_{\mathrm{s}}=0.65, p=0.007, N=16\right)$. For rotating illusions, vection onset also correlated negatively with vection magnitude $\left(\mathrm{r}_{\mathrm{s}}=0.67, p=0.005, N=17\right)$.

\section{Discussion}

Firstly, the present study aimed to investigate if AVGPs perceived more motion in optimised Fraser Wilcox illusions when presented using VR headsets compared to NVGPs. Stronger perception of the illusion was expected based on their improved perceptual skills reported in several previous studies (e.g. Chopin et al. 2019; Li et al. 2009, 2010). Contrary to predictions, no difference in illusion strength ratings was found between AVGPs and NVGPs. A follow-up study conducted online using both grey scaled as well as colourful versions of the Fraser Wilcox illusions confirmed these results, finding no difference in illusion strength ratings between AVGPs and NVGPs (for details, see Supplementary material).

Secondly, the experience of vection when viewing the illusions was of interest. The sensation of illusory self-motion has been linked to attentional and cognitive processes and is believed to decrease with increased cognitive or attentional load (Seno et al. 2011). Playing action video games has been shown to improve attentional skills (Bavelier and Green 2019; Föcker et al. 2018, 2019) and therefore, a stronger experience of vection in individuals that spend extensive time playing first person shooter games was expected and found in this experiment.

Finally, this study aimed to investigate whether AVGPs would experience less discomfort in the virtual environment compared to their non-video game playing counterparts. Due to their significant experience with virtual environments, AVGPs are believed to experience less discomfort in VR in general based on adaptation and habituation processes (Domeyer et al. 2013; Hill \& Haworth, 200; Keshavarz 2016). This notion was confirmed by the current study as AVGPs experienced less discomfort while looking at the motion illusions in VR compared to NVGPs.

Our findings showed that based on self-reports, there were no differences in illusion strength perceived in the visual motion illusion between gamers and non-gamers. The lack of group differences in the perception of motion strength was rather unexpected given that previous literature has pointed to the enhanced perceptual abilities in gamers which were expected to be linked to an increased susceptibility to optimised Fraser Wilcox illusions (e.g. Chopin et al. 2019; Hutchinson and Stocks 2013).

Table 2 Spearmen correlation of illusion strength ratings, vection measures (magnitude, onset, duration) and discomfort ratings for expanding and rotating illusions for AVGPs

\begin{tabular}{|c|c|c|c|c|c|c|c|c|}
\hline & \multicolumn{4}{|l|}{ Rotating } & \multicolumn{4}{|c|}{ Expanding } \\
\hline & $\begin{array}{l}\text { Illusion } \\
\text { strength }\end{array}$ & $\begin{array}{l}\text { Vection } \\
\text { magnitude }\end{array}$ & $\begin{array}{l}\text { Vection } \\
\text { onset }\end{array}$ & $\begin{array}{l}\text { Vection } \\
\text { duration }\end{array}$ & $\begin{array}{l}\text { Illusion } \\
\text { strength }\end{array}$ & $\begin{array}{l}\text { Vection } \\
\text { magnitude }\end{array}$ & $\begin{array}{l}\text { Vection } \\
\text { onset }\end{array}$ & $\begin{array}{l}\text { Vection } \\
\text { duration }\end{array}$ \\
\hline $\begin{array}{l}\text { Vection } \\
\text { magnitude }\end{array}$ & $0.66^{* * *}$ & - & & & $0.71 * * *$ & - & & \\
\hline Vection onset & -0.37 & -0.43 & - & & -0.26 & -0.39 & - & \\
\hline Vection duration & 0.35 & $0.48 *$ & $-0.52 *$ & - & -0.03 & 0.32 & -0.24 & - \\
\hline Discomfort & $0.52 *$ & 0.29 & -0.10 & 0.04 & $0.43 *$ & $0.44 *$ & -0.32 & -0.03 \\
\hline
\end{tabular}

${ }^{*} p<0.05 ; * *<<0.01 ; * * * p<0.001, N=22, N_{\text {Onset }}=20, N_{\text {Duration }}=20$ 
In a recent study, He et al. (2020) found that individuals with better contrast discrimination perceived faster illusory motion in rotating illusions. Enhanced contrast sensitivity has also been observed as a result of video game training with AVGPs outperforming non-gamers in identifying Gabor patches with varying contrast ( $\mathrm{Li}$ et al. 2009), which again would suggest that the AVGPs in our study should have perceived more illusory motion in the presented images compared to NVGPs. However, differences in contrast sensitivity and discrimination thresholds between gamers and nongamers could be expected but were not measured in the current study; therefore, it cannot be concluded that the AVGPs in this study actually had lower contrast discrimination thresholds. Follow-up studies should include a measure of contrast discrimination prior to the experiment to control 'baseline perceptual skills' in gamers and non-gamers.

Illusory motion in the He et al. (2020) study was measured by adding physical rotation in the opposite direction of the illusory rotation to the stimulus and determining the physical speed required to cancel out the illusion (He et al. 2020). In the current study, however, the strength of the illusion was rated on a scale from 0 to 10 after the illusion was presented, with 0 representing no motion at all and 10 representing the strongest perception of illusory motion. The way illusion strength was measured in the current study might not have been sensitive enough to pick up differences in experienced illusion strength between gamers and non-gamers. An additional difference between the current study and the experiment conducted by He et al. (2020) was the type of viewing condition. In the current experiment, a free viewing condition was applied, meaning that participants did not have to focus on a specific point could move their eyes over the image trying to elicit the strongest perception of illusory motion. In the He et al. (2020) study, however, participants focused on a fixation dot with the illusion presented to the side of it in the participant's peripheral FOV. The free viewing condition applied in the current study might have affected the experience of illusion strength for gamers and non-gamers differently.

A possible difference in the perception of the motion illusion between gamers and non-gamers that was not measured in the current study could be the duration, onset and offset of the illusion rather than the perceived strength of the motion. Illusory motion perceived in these types of illusions tends to stop after a few seconds and start again after a 'recovery phase' (Tomimatsu et al. 2011). AVGPs could have perceived the illusory motion for a longer period of time and/or had shorter onset times and perceived the illusion quicker compared to NVGPs. Therefore, further research should include measures of illusion onset and offset. In line with predictions, AVGPs experienced a stronger sensation of vection which suggests that the experience of vection is not merely a bottom-up process and influenced by stimulus properties (size, speed) but also requires some form of cognitive processing 'power' (Seno et al. 2011). Playing action video games improves attentional control functions, meaning that the gamers in this experiment experience less cognitive load and therefore more vection compared to NVGPs.

An alternative explanation for AVGPs experiencing more vection compared to NVGPs could be an increased FOV and better global perception obtained through video game play (Buckley et al. 2010). The perception of vection increases with an increase of FOV, with larger displays or moving stimuli covering larger parts of the FOV resulting in a stronger experience of illusory self-motion (e.g. Allison et al. 1999; Keshavarz et al. 2017). This could partly explain the stronger experience of vection found for AVGPs in the current study. However, larger FOVs are also related or often cause a stronger sensation of VR sickness (e.g. Adhanom et al. 2020; Fernandes and Feiner 2016; Al Zayer et al. 2019). AVGPs in this study, however, experienced less discomfort compared to NVGPs, which would in turn contradict the notion of AVGPs obtaining larger FOV due to excessive video game play.

Differences in the experience of vection between the gamer groups were only found for the magnitude of vection, but not for vection onset or duration times. This suggests that cognitive load might primarily affect the strength of the experienced vection rather than the temporal aspect of the experience. The three vection measures are likely to represent different processes involved during vection, suggesting that previous experience with video games modulates the magnitude of the experienced vection due to improved attentional and cognitive skills but does not affect when and for how long this sensation is experienced (Seno et al. 2017).

The relationship between vection and discomfort experienced in virtual environments is rather complex with vection and discomfort often occurring together (Hettinger et al. 1990; Plouzeau et al. 2015), resulting in vection being associated with adverse symptoms experienced in VR. However, vection can also be a positive aspect of VR. The player's experience of VR can be improved through vection as it represents a more realistic sensation of self-motion (Riecke 2011). Virtual environments that elicit more intense experiences of vection are often more immersive and lead to stronger feelings of presence (Weech et al. 2019). Presence is the feeling and belief of being in and being part of the virtual environment, reacting to stimuli as if they were in the real world (Heeter 1992). Presence has also been related to the experience of discomfort in VR, with vection often seen as the link between these two phenomena (Weech et al. 2019). In an ideal VR experience, presence and vection would be present with minimised or no discomfort. Habituation of individuals to virtual environments might help create these ideal VR experiences. Participants in this study that had excessive experience with action video games experienced more vection while suffering from less adverse symptoms. As mentioned above, increases in the 
vection experience could be related to the improved cognitive skills of AVGPs but could also be related to the abundant experience with virtual environments. Bubka et al. (2008) found that individuals experience more vection for motion directions that they are more used to, in their study they compared forward and backward self-motion and found stronger vection for forward self-motion basing these findings on the stronger experience with real self-motion of the observers. AVGPs have more experience with locomotion through virtual environments which in turn could partly explain the stronger experience of vection in the current study.

Again, as predicted, AVGPs experienced less discomfort while viewing the illusions in VR compared to the NVGPs in this study. This difference in experienced discomfort can be explained by their significant experience with virtual environments. They experienced less discomfort in VR based on adaptation and habituation processes (Domeyer et al. 2013; Hill \& Haworth, 200; Keshavarz 2016). To our knowledge, so far, adaptation effects have only been investigated by exposing the individual to the same sickness-inducing environment. Individuals experienced the same real or virtual environment (e.g. sea voyage, driving simulator, flight simulator) repeatedly or for long periods which in turn led to decreases in adverse symptoms (e.g. Keshavarz 2016; Li et al. 2012; Reason 1978). To our knowledge, the current study is the first to investigate if adaptation effects to discomfort in VR can be gained with repeated exposure to virtual environments in action video games. Do individuals that spend long periods of time playing video games on the computer or console build up habituation to virtual environments that can translate to a virtual environment presented in a VR headset? AVGPs and NVGPs in this study did not differ in their experience with VR headsets; rather, AVGPs gained their experience with virtual environments using different devices. This can be of interest for individuals that are more susceptible to experience discomfort in virtual environments as adaptation can be time consuming and requires high motivation from the individual suffering from discomfort. An individual that experiences severe adverse symptoms when using a VR headset for the first time might not be willing to expose themselves to this uncomfortable experience again. Instead of using the exact stimulus and display type to train and build up habituation in these more susceptible individuals, a less nauseating set up could be used. Additionally, individual differences in the experience of discomfort in VR could be taken into account when designing VR applications or games. They could include tutorials that enable the user to experience VR in a less sickness-inducing environment first and slowly increase the sickness inducing stimuli (for example to speed of motion) to slowly allow the user to adapt to their surroundings and adapt.

Immersion and the level of presence experienced by an individual might also affect their experience of discomfort in VR (Weech et al. 2019). The current study did not measure the level of immersion or presence of the participants; however, presence seems to be related to the experience of vection, with an individual that is feeling more immersed in the virtual environment also generally experiencing more vection (Riecke et al. 2006; Weech et al. 2019). The relationship of VR sickness and presences is still unclear, with several studies finding a positive relationship between them (Busscher et al. 2011; Liu and Uang 2011; Ling et al. 2013) and with several others finding the opposite effect (Cooper et al. 2015; Nichols et al. 2000; Kim et al. 2005; Witmer and Singer 1998). A positive relationship between the two phenomena would suggest that based on the increased experience of vection by the AVGPs in the current study they should have also experienced more discomfort. However, the opposite effect was found in the current study, supporting the notion of a negative relationship between presence and VR sickness. A follow-up study could additionally measure immersion and presence in AVGPS and NVGPs to further investigate the relationship of vection and presence as well as their effects on VR sickness.

The correlation results revealed that the relationship between the vection measures seems to be driven mainly by vection duration times and was stronger for NVGPs particularly for expanding motion illusions.

This might suggest that for NVGPs in this study, the timing of vection was more related to the perceived motion in the stimuli (particularly for expanding illusions) compared to AVGPs, indicating that NVGPs could be more sensitive to changes in vection onset and duration induced by perceived motion compared to AVGPs. The relationship of illusion strength and vection measures also seems to differ between rotating and expanding illusions for NVGP. Illusion strength for rotating stimuli did not correlate with any vection measure whereas for expanding illusions vection magnitude and duration showed a significant relationship with illusion strength. For AVGPs, however, no difference in the relationship of illusion strength and vection or discomfort between expanding and rotating illusions can be found. These findings would suggest that for NVGPs the temporal experience of vection is dependent on the direction of perceived motion (lateral vs. motion in depth). The intensity of perceived motion in depth related to the temporal experience of vection whereas the intensity of perceived rotating motion was not related to the experience of vection. These findings would suggest that there is a difference in the perception of the illusory motion between NVGPs and AVGPs; this difference might not be reflected by differences in illusion strength but by the differing relations between illusion strength, vection and discomfort found for the two gamer groups.

When looking at the correlation results, we also found that for AVGPs illusion strength was positively correlated with vection magnitude and discomfort whereas for NVGPs vection magnitude and vection duration times significantly 
correlated with illusion strength only for expanding motion illusions. The stronger the movement perceived in the illusion, the more discomfort AVGPs experienced, suggesting that the discomfort was caused by the contrast illusions rather than the virtual environment. This could be explained by the link between playing video games and the severity of headaches associated with migraine (Di Luzio et al. 2021). Individuals suffering from migraines often report increased symptoms when exposed to contrast striped patterns (similar to the illusions presented in this study; e.g. Shepherd 2000). Interindividual differences in the perception of motion illusions have been observed in individuals with migraine (Harle et al. 2006; Shepherd 2000; Shepherd et al. 2013), with self-report measures revealing that individuals with migraine show a higher susceptibility to motion patterns compared to neurotypical observers especially for high-contrast stationary images (He et al. 2020). This elevated pattern sensitivity in migraine can also be linked to improved contrast detection and prolonged motion aftereffects. However, it is important to note that He et al. (2020) showed that individuals with better contrast discrimination tended to perceive the illusions as moving faster regardless of migraine status. Enhanced contrast sensitivity has also been observed as a result of video game training ( $\mathrm{Li}$ et al. 2009). In this experiment, gamers were asked if a Gabor patch varying in contrast occurs in the first or the second time intervals of a single trial. Individuals trained on action video games outperform participants in the control group on this task showing enhanced contrast sensitivity ( $\mathrm{Li}$ et al. 2009). Additionally, a link between hours spent playing first-person shooter games and migraine symptoms was found which could explain the positive relationship between illusions strength ratings and discomfort found for the AVGPs in this study (Di Luzio et al. 2021). The relationship between contrast sensitivity, migraine and video gaming should be further investigated to assess its influence on the perception of illusory motion. Migraine status of participants should be assessed, and their contrast sensitivity thresholds should be determined. This would allow for a comparison between gamers that show migraine symptoms and gamers that show no symptoms.

However, the results of this study need to be interpreted with caution; analysis with likelihood ratio tests for all significant effects found in this study showed that the strength of these effects were minimal. Model fit for full models and null models was compared in these tests resulting in negative BIC values indicating support for the null model over the full model. These negative BIC values suggest that the strength of evidence for the effect gamer type on the discomfort ratings was fairly small. The results of this study should be replicated with gamer groups equal for sex and age (e.g. Arns and Cerney 2005; Chaumillon et al. 2017; Curry et al., Munafo et al. 2017).
The differences in discomfort we found between gamers and non-gamers in this study could be affected by the uneven distribution of gender in the groups. The AVGP group consisted mainly of males and only a limited number of females ( 15 males, 7 females), whereas the NVGP group mainly consisted of females ( 4 males, 17 females). Females are believed to be more susceptible to motion sickness and discomfort experienced in real life scenarios, such as traveling on a bus (Turner and Griffin 1999) as well as in driving simulators (e.g. Chaumillon et al. 2017; Schweig et al. 2018) and virtual environments presented using HMDs (e.g. Curry et al. 2020). Research investigating the effect of motion sickness susceptibility or an individual's motion sickness history, however, found that when susceptibility was kept constant between males and females no difference in VR sickness was found between genders (Stanney et al. 2003). This would suggest that rather than gender NVGPs, predisposition to motion sickness could explain the differences in discomfort between the gamer groups. Individuals that are more prone to experience motion sickness-like symptoms might also be less likely to pick up gaming as a hobby, suggesting that AVGPs do not experience less discomfort due to their increased exposure to virtual environments when playing video games, but rather that these individuals were predisposed to experience less discomfort in general which is why they picked up gaming in the first instance. To avoid gender or susceptibility to motion sickness affecting group comparisons instead of recruiting AVGPs and NVGPs, naive participants could be trained using action video games or other types of video games to further investigate the beneficial effect of gaming on vection and discomfort.

Additionally, it might be argued that rating scales are not an adequate instrument to compare gamers and non-gamers due to the confound with verbal assessment. However, as gamers did not differ from non-gamers 'by default' across all rating scales, e.g. gamers did not differ in motion illusion strength (which was assessed by subjective rating), but they differ in perceived discomfort (which was also assessed by subjective raring), we would argue that the verbal assessment is a justified instrument to explore discomfort and differences between both groups. Furthermore, as discomfort is a subjective feeling, it can be only directly tested by asking participants to indicate their subjective impression of discomfort. However, future studies should include measurements such as the onset of motion illusions which can be measured by button press. Illusion onset and offset were not measured in this study due to participants already indicating vection onset and duration by button press.

The results of this study showed that illusion strength ratings are not sensitive enough to indicate differences in the perception of the illusions between gamers and non-gamers. Including onset, duration and offset measures for illusory motion would allow us to further investigate the relationship 
between contrast sensitivity and susceptibility to illusory motion elicited by optimised Fraser Wilcox illusions (Keshavarz et al. 2017; Riecke et al. 2006; Seno et al. 2017). Onset times of the motion illusion would be expected to be shorter for individuals with higher contrast sensitivity. A different measure of illusion strength could also be applied to investigate the effect of contrast sensitivity. Instead of using ratings to report illusion strength, a 'nulling' method similar to the one used by $\mathrm{He}$ and colleagues could be applied. The illusory motion can be measured quantitatively by adding physical rotation into the test stimulus and determining the speed required to cancel out the illusory motion (e.g. He et al. 2020; Hisakata and Murakami 2008; Thornton and Zdravković 2020). This method was not chosen for the current experiment as it has only been applied to rotating but not expanding illusory motion. Although no differences between expanding and rotating illusions were found in this experiment, we aimed to investigate whether both motion directions were able to elicit vection in the observer.

To summarise, future studies will benefit from more objective measurements of baseline perceptual skills in gamers, e.g. contrast sensitivity, testing motion perception to physical stimuli as well as the onset of motion illusions. Furthermore, training designs need to be included in order to understand the causal relationship between gaming, perception of motion illusion and discomfort. For instance, it might be argued that even though gamers do not perceive motion illusions as stronger in the current study, they might have experienced a shorter illusion onset and longer illusion duration. In order to measure the onset of motion illusions, in further follow-up experiments, participants will be asked to indicate via button press as soon as they perceive the static objects to be moving. Furthermore, follow-up experiments should include the measurement of contrast sensitivity in gamers and non-gamers. Given previous research, gamers should show a higher contrast sensitivity compared to nongamers which might explain any perceptual differences in the populations.

In conclusion, the current study showed that illusory motion is efficient in eliciting vection and no explicit physical motion is necessary. It also demonstrated that AVGPs are more susceptible to vection elicited by motion illusions, confirming the notion that vection is affected by cognitive and attentional load as AVGPs have been shown to have better attentional control. AVGPs also experienced less discomfort overall compared to NVGPs which could be explained by them having more experience with virtual environments and therefore having adapted to the sickness-inducing aspects of such environments. Additionally, the discomfort experienced by AVGPs was positively correlated with illusion strength, suggesting that the discomfort that they experienced was caused by the contrast illusions rather than the virtual environment itself.

Supplementary Information The online version contains supplementary material available at https://doi.org/10.1007/s41465-021-00215-6.

Acknowledgements The authors would like to thank Foivos Vantzos for his technical support in designing the VR application.

Authors' Contribution K.P. and J.F. conceived the study; K.P., L.O.H., J.F. and P.D. designed the experiment; K.P. performed the experiment; K.P. analysed the data; and K.P., L.O.H., J.F., P.D. and A.P. wrote the paper.

Funding Katharina Pöhlmann received a scholarship from the School of Psychology at the University of Lincoln.

Data Availability Associated data can be downloaded from https://doi. org/10.6084/m9.figshare. 13398458

\section{Declaration}

Conflict of Interest The authors declare that they have no conflict of interest.

Open Access This article is licensed under a Creative Commons Attribution 4.0 International License, which permits use, sharing, adaptation, distribution and reproduction in any medium or format, as long as you give appropriate credit to the original author(s) and the source, provide a link to the Creative Commons licence, and indicate if changes were made. The images or other third party material in this article are included in the article's Creative Commons licence, unless indicated otherwise in a credit line to the material. If material is not included in the article's Creative Commons licence and your intended use is not permitted by statutory regulation or exceeds the permitted use, you will need to obtain permission directly from the copyright holder. To view a copy of this licence, visit http://creativecommons.org/licenses/by/4.0/.

\section{References}

Adhanom, I. B., Griffin, N. N., MacNeilage, P., \& Folmer, E. (2020). The effect of a foveated field-of-view restrictor on VR sickness. In 2020 IEEE conference on virtual reality and $3 D$ user interfaces (VR) (pp. 645-652). IEEE.

Akiduki, H., Nishiike, S., Watanabe, H., Matsuoka, K., Kubo, T., \& Takeda, N. (2003). Visual-vestibular conflict induced by virtual reality in humans. Neuroscience Letters, 340(3), 197-200.

Al Zayer, M., Adhanom, I. B., MacNeilage, P., \& Folmer, E. (2019). The effect of field-of-view restriction on sex bias in vr sickness and spatial navigation performance. In Proceedings of the 2019 CHI Conference on Human Factors in Computing Systems (pp. 1-12).

Allison, R. S., Howard, I. P., \& Zacher, J. E. (1999). Effect of field size, head motion, and rotational velocity on roll vection and illusory selftilt in a tumbling room. Perception, 28(3), 299-306.

Anguera, J. A., Boccanfuso, J., Rintoul, J. L., Al-Hashimi, O., Faraji, F., Janowich, J., Kong, E., Larraburo, Y., Rolle, C., Johnston, E., \& Gazzaley, A. (2013). Video game training enhances cognitive control in older adults. Nature, 501(7465), 97-101. 
Arcioni, B., Palmisano, S., Apthorp, D., \& Kim, J. (2019). Postural stability predicts the likelihood of cybersickness in active HMD-based virtual reality. Displays, 58, 3-11.

Arns, L. L., \& Cerney, M. M. (2005). The relationship between age and incidence of cybersickness among immersive environment users. In IEEE Proceedings. VR 2005. Virtual Reality, 2005. (pp. 267-268). IEEE.

Backus, B. T., \& Oruç, I. (2005). Illusory motion from change over time in the response to contrast and luminance. Journal of Vision, 5(11), $10-10$.

Bates, D., Mächler, M., Bolker, B., \& Walker, S. (2014). Fitting linear mixed-effects models using lme4. arXiv preprint arXiv:1406.5823.

Bavelier, D. (2019) Video game playing questionnaire. https://www. unige.ch/fapse/brainlearning/vgq. Accessed 15 Nov 2019.

Bavelier, D., \& Green, C. S. (2019). Enhancing attentional control: lessons from action video games. Neuron, 104(1), 147-163.

Bavelier, D., Achtman, R. L., Mani, M., \& Föcker, J. (2012). Neural bases of selective attention in action video game players. Vision Research, 61, 132-143.

Bediou, B., Adams, D. M., Mayer, R. E., Tipton, E., Green, C. S., \& Bavelier, D. (2018). Meta-analysis of action video game impact on perceptual, attentional, and cognitive skills. Psychological Bulletin, 144(1), 77

Bejjanki, V. R., Zhang, R., Li, R., Pouget, A., Green, C. S., Lu, Z. L., \& Bavelier, D. (2014). Action video game play facilitates the development of better perceptual templates. Proceedings of the National Academy of Sciences, 111(47), 16961-16966.

Berchtold, A. (2010). Sequence analysis and transition models. In M Breed, J Moore (eds.), Encyclopedia of Animal Behavior, pp. 139145. Academic Press, Oxford. https://doi.org/10.1016/b978-0-08045337-8.00233-3.

Berthoz, A., Pavard, B., \& Young, L. R. (1975). Perception of linear horizontal self-motion induced by peripheral vision (linear vection). Basic characteristics and visual vestibular interactions. Experimental Brain Research, 23, 471-489.

Bonato, F., Bubka, A., Palmisano, S., Phillip, D., \& Moreno, G. (2008). Vection change exacerbates simulator sickness in virtual environments. Presence Teleoperators and Virtual Environments, 17(3), 283-292.

Boot, W. R., Kramer, A. F., Simons, D. J., Fabiani, M., \& Gratton, G. (2008). The effects of video game playing on attention, memory, and executive control. Acta Psychologica, 129(3), 387-398.

Brandt, T., Dichgans, J., \& Koenig, E. (1973). Differential effects of central versus peripheral vision on egocentric and exocentric motion perception. Experimental Brain Research, 16, 476-491.

Breheny, P., \& Burchett, W. (2017). Visualization of regression models using visreg. $R J, 9(2), 56$.

Bubka, A., Bonato, F., \& Palmisano, S. (2008). Expanding and contracting optic-flow patterns and vection. Perception, 37(5), 704-711.

Buckley, D., Codina, C., Bhardwaj, P., \& Pascalis, O. (2010). Action video game players and deaf observers have larger Goldmann visual fields. Vision Research, 50(5), 548-556.

Busscher, B., de Vliegher, D., Ling, Y., \& Brinkman, W. P. (2011). Physiological measures and self-report to evaluate neutral virtual reality worlds. Journal of Cybertherapy and Rehabilitation, 4(1), $15-25$.

Chaumillon, R., Romeas, T., Paillard, C., Bernardin, D., Giraudet, G., Bouchard, J. F., \& Faubert, J. (2017). The use of transdermal scopolamine to solve methodological issues raised by gender differences in susceptibility to simulator sickness. Transportation Research Part F: Traffic Psychology and Behaviour, 47, 42-58.

Chisholm, J. D., \& Kingstone, A. (2012). Improved top-down control reduces oculomotor capture: the case of action video game players. Attention, Perception, \& Psychophysics, 74(2), 257-262.
Chisholm, J. D., Hickey, C., Theeuwes, J., \& Kingstone, A. (2010). Reduced attentional capture in action video game players. Attention, Perception, \& Psychophysics, 72(3), 667-671.

Chopin, A., Bediou, B., \& Bavelier, D. (2019). Altering perception: the case of action video gaming. Current Opinion in Psychology, 29, $168-173$.

Conway, B. R., Kitaoka, A., Yazdanbakhsh, A., Pack, C. C., \& Livingstone, M. S. (2005). Neural basis for a powerful static motion illusion. Journal of Neuroscience, 25(23), 5651-5656.

Cooper, N., Milella, F., Cant, I., Pinto, C., White, M., \& Meyer, G. (2015). The effects of multisensory cues on the sense of presence and task performance in a virtual reality environment.

Curry, C., Li, R., Peterson, N., \& Stoffregen, T. A. (2020). Cybersickness in virtual reality head-mounted displays: examining the influence of sex differences and vehicle control. International Journal of Human Computer Interaction, 36(12), 1161-1167.

Di Luzio, P., Borgomaneri, S., Sanchioni, S., Tessari, A., \& Romei, V. (2021). Exposure to first-person shooter videogames is associated with multisensory temporal precision and migraine incidence. Cortex, 134, 223-238.

Dichgans, J., \& Brandt, T. (1978). Visual-vestibular interaction: effects on self-motion perception and postural control. In Perception (pp. 755-804). Springer.

Diels, C., Ukai, K., \& Howarth, P. A. (2007). Visually induced motion sickness with radial displays: effects of gaze angle and fixation. Aviation, Space, and Environmental Medicine, 78(7), 659-665.

Domeyer, J. E., Cassavaugh, N. D., \& Backs, R. W. (2013). The use of adaptation to reduce simulator sickness in driving assessment and research. Accident Analysis \& Prevention, 53, 127-132.

Faubert, J., \& Herbert, A. M. (1999). The peripheral drift illusion: a motion illusion in the visual periphery. Perception, 28(5), 617-621.

Fernandes, A. S., \& Feiner, S. K. (2016). Combating VR sickness through subtle dynamic field-of-view modification. In 2016 IEEE symposium on $3 D$ user interfaces (3DUI) (pp. 201-210). IEEE.

Fischer, M. H., \& Kornmüller, A. E. (1930). Der Schwindel. In Handbuch der normalen und pathologischen Physiologie (pp. 442-494). Springer.

Föcker, J., Cole, D., Beer, A. L., \& Bavelier, D. (2018). Neural bases of enhanced attentional control: lessons from action video game players. Brain and Behavior, 8(7), e 01019.

Föcker, J., Mortazavi, M., Khoe, W., Hillyard, S. A., \& Bavelier, D. (2019). Neural correlates of enhanced visual attentional control in action video game players: an event-related potential study. Journal of Cognitive Neuroscience, 31(3), 377-389.

Franceschini, S., Trevisan, P., Ronconi, L., Bertoni, S., Colmar, S., Double, K., Facoetti, A., \& Gori, S. (2017). Action video games improve reading abilities and visual-to-auditory attentional shifting in English-speaking children with dyslexia. Scientific Reports, 7(1), $1-12$.

Fraser, A., \& Wilcox, K. J. (1979). Perception of illusory movement. Nature, 281(5732), 565-566.

Green, C. S., \& Bavelier, D. (2006). Effect of action video games on the spatial distribution of visuospatial attention. Journal of Experimental Psychology: Human Perception and Performance, $32(6), 1465$

Green, C. S., Pouget, A., \& Bavelier, D. (2010). Improved probabilistic inference as a general learning mechanism with action video games. Current Biology, 20(17), 1573-1579.

Harle, D. E., Shepherd, A. J., \& Evans, B. J. (2006). Visual stimuli are common triggers of migraine and are associated with pattern glare. Headache: The Journal of Head and Face Pain, 46(9), 1431-1440.

Harrell Jr, F. E., \& Harrell Jr, M. F. E. (2019). Package 'Hmisc'. CRAN2018, 2019, 235-6.

He, C., Nguyen, B. N., Chan, Y. M., \& McKendrick, A. M. (2020). Illusory motion perception is associated with contrast discrimination but not motion sensitivity, self-reported visual discomfort, or 
migraine status. Investigative Ophthalmology \& Visual Science, 61(8), 43-43.

Heeter, C. (1992). Being there: the subjective experience of presence. Presence Teleoperators and Virtual Environments, 1(2), 262-271.

Hermens, F., \& Zanker, J. (2012). Looking at Op Art: gaze stability and motion illusions. i-Perception, 3(5), 282-304.

Hettinger, L. J., Berbaum, K. S., Kennedy, R. S., Dunlap, W. P., \& Nolan, M. D. (1990). Vection and simulator sickness. Military Psychology, 2(3), 171-181.

Heutink, J., Broekman, M., Brookhuis, K. A., Melis-Dankers, B. J., \& Cordes, C. (2019). The effects of habituation and adding a restframe on experienced simulator sickness in an advanced mobility scooter driving simulator. Ergonomics, 62(1), 65-75.

Hill, K. J., \& Howarth, P. A. (2000). Habituation to the side effects of immersion in a virtual environment. Displays, 21(1), 25-30.

Hisakata, R., \& Murakami, I. (2008). The effects of eccentricity and retinal illuminance on the illusory motion seen in a stationary luminance gradient. Vision Research, 48(19), 1940-1948.

Hoffman, D. M., Girshick, A. R., Akeley, K., \& Banks, M. S. (2008). Vergence-accommodation conflicts hinder visual performance and cause visual fatigue. Journal of Vision, 8(3), 33-33.

Howarth, P. A., \& Hodder, S. G. (2008). Characteristics of habituation to motion in a virtual environment. Displays, 29(2), 117-123.

Hutchinson, C. V., \& Stocks, R. (2013). Selectively enhanced motion perception in core video gamers. Perception, 42(6), 675-677.

Kass, R. E., \& Wasserman, L. (1995). A reference Bayesian test for nested hypotheses and its relationship to the Schwarz criterion. Journal of the American Statistical Association, 90(431), 928-934.

Kennedy, R. S., Stanney, K. M., \& Dunlap, W. P. (2000). Duration and exposure to virtual environments: sickness curves during and across sessions. Presence Teleoperators and Virtual Environments, 9(5), $463-472$.

Keshavarz, B. (2016). Exploring behavioral methods to reduce visually induced motion sickness in virtual environments. In International Conference on Virtual, Augmented and Mixed Reality (pp. 147155). Springer.

Keshavarz, B., Riecke, B. E., Hettinger, L. J., \& Campos, J. L. (2015). Vection and visually induced motion sickness: how are they related? Frontiers in Psychology, 6, 472.

Keshavarz, B., Speck, M., Haycock, B., \& Berti, S. (2017). Effect of different display types on vection and its interaction with motion direction and field dependence. i-Perception, 8(3), 2041669517707768.

Kim, Y. Y., Kim, H. J., Kim, E. N., Ko, H. D., \& Kim, H. T. (2005). Characteristic changes in the physiological components of cybersickness. Psychophysiology, 42(5), 616-625.

Kitaoka, A. (2006). Anomalous motion illusion and stereopsis. J Three Dimensional Images (Tokyo), 20, 9-14.

Kitaoka, A., \& Ashida, H. (2003). Phenomenal characteristics of the peripheral drift illusion. Vision, 15(4), 261-262.

Kramida, G. (2015). Resolving the vergence-accommodation conflict in head-mounted displays. IEEE Transactions on Visualization and Computer Graphics, 22(7), 1912-1931.

Krueger, C., \& Tian, L. (2004). A comparison of the general linear mixed model and repeated measures ANOVA using a dataset with multiple missing data points. Biological Research for Nursing, 6(2), 151157.

Kuznetsova, A., Brockhoff, P. B., \& Christensen, R. H. (2017). lmerTest package: tests in linear mixed effects models. Journal of Statistical Software, 82(13), 1-26.

Lenth, R., Singmann, H., Love, J., Buerkner, P., \& Herve, M. (2018). Emmeans: estimated marginal means, aka least-squares means. $\mathrm{R}$ package version, 1(1), 3 .

Li, R., Polat, U., Makous, W., \& Bavelier, D. (2009). Enhancing the contrast sensitivity function through action video game training. Nature Neuroscience, 12(5), 549-551.
Li, R., Polat, U., Scalzo, F., \& Bavelier, D. (2010). Reducing backward masking through action game training. Journal of Vision, 10(14), 33-33.

Li, R. W., Ngo, C., Nguyen, J., \& Levi, D. M. (2011). Video-game play induces plasticity in the visual system of adults with amblyopia. PLoS Biology, 9(8), e1001135.

Li, J. R., Zhu, L., Yuan, W., Jin, G. R., \& Sun, J. J. (2012). Habituation of seasickness in adult during a long voyage. Zhonghua er bi yan hou tou jing wai ke za zhi= Chinese journal of otorhinolaryngology head and neck surgery, 47(8), 642.

Ling, Y., Nefs, H. T., Brinkman, W. P., Qu, C., \& Heynderickx, I. (2013). The relationship between individual characteristics and experienced presence. Computers in Human Behavior, 29(4), 1519-1530.

Liu, C. L., \& Uang, S. T. (2011). Effects of presence on causing cybersickness in the elderly within a $3 \mathrm{D}$ virtual store. In International conference on human-computer interaction (pp. 490-499). Springer.

Luke, S. G. (2017). Evaluating significance in linear mixed-effects models in R. Behavior Research Methods, 49(4), 1494-1502.

Moss, J. D., \& Muth, E. R. (2011). Characteristics of head-mounted displays and their effects on simulator sickness. Human Factors, 53(3), 308-319.

Munafo, J., Diedrick, M., \& Stoffregen, T. A. (2017). The virtual reality head-mounted display Oculus Rift induces motion sickness and is sexist in its effects. Experimental Brain Research, 235(3), 889-901.

Murakami, I., Kitaoka, A., \& Ashida, H. (2006). A positive correlation between fixation instability and the strength of illusory motion in a static display. Vision Research, 46(15), 2421-2431.

Nakamura, S. (2006). Effects of depth, eccentricity and size of additional static stimulus on visually induced self-motion perception. Vision Research, 46(15), 2344-2353.

Nakamura, S. (2013). The minimum stimulus conditions for vectiontwo-and four-stroke apparent motions can induce self-motion perception. Perception, 42(2), 245-247.

Nakamura, S., \& Shimojo, S. (1999). Critical role of foreground stimuli in perceiving visually induced self-motion (vection). Perception, 28(7), 893-902.

Naor-Raz, G., \& Sekuler, R. (2000). Perceptual dimorphism in visual motion from stationary patterns. Perception, 29(3), 325-335.

Nichols, S., Haldane, C., \& Wilson, J. R. (2000). Measurement of presence and its consequences in virtual environments. International Journal of Human-Computer Studies, 52(3), 471-491.

Otero-Millan, J., Macknik, S. L., \& Martinez-Conde, S. (2012). Microsaccades and blinks trigger illusory rotation in the "rotating snakes" illusion. Journal of Neuroscience, 32(17), 6043-6051.

Palmisano, S., \& Chan, A. Y. (2004). Jitter and size effects on vection are immune to experimental instructions and demands. Perception, 33(8), 987-1000.

Palmisano, S., Bonato, F., Bubka, A., \& Folder, J. (2007). Vertical display oscillation effects on forward vection and simulator sickness. Aviation, Space, and Environmental Medicine, 78(10), 951-956.

Palmisano, S., Mursic, R., \& Kim, J. (2017). Vection and cybersickness generated by head-and-display motion in the Oculus Rift. Displays, $46,1-8$.

Pavan, A., Boyce, M., \& Ghin, F. (2016). Action video games improve direction discrimination of parafoveal translational global motion but not reaction times. Perception, 45(10), 1193-1202.

Plouzeau, J., Paillot, D., Chardonnet, J. R., \& Merienne, F. (2015). Effect of proprioceptive vibrations on simulator sickness during navigation task in virtual environment. In: International Conference on Artificial Reality and Telexistence Eurographics Symposium on Virtual Environments, Japon, 2015-10-28 - International Conference on Artificial Reality and Telexistence Eurographics Symposium on Virtual Environments - 2015.

Reason, J. T. (1978). Motion sickness adaptation: a neural mismatch model. Journal of the Royal Society of Medicine, 71(11), 819-829. 
Regan, C. (1995). An investigation into nausea and other side-effects of head-coupled immersive virtual reality. Virtual Reality, 1(1), 17-31.

Riecke, B. E. (2011). Compelling self-motion through virtual environments without actual self-motion: using self-motion illusions ("vection") to improve user experience in VR. Virtual Reality, 149-176. https://doi.org/10.5772/13150.

Riecke, B. E., Västfjäll, D., Larsson, P., \& Schulte-Pelkum, J. (2005). Top-down and multi-modal influences on self-motion perception in virtual reality. In Proceedings of HCI international 2005 (pp. 1-10).

Riecke, B. E., Schulte-Pelkum, J., Avraamides, M. N., Heyde, M. V. D., \& Bülthoff, H. H. (2006). Cognitive factors can influence selfmotion perception (vection) in virtual reality. ACM Transactions on Applied Perception (TAP), 3(3), 194-216.

Riesenhuber, M. (2004). An action video game modifies visual processing. Trends in Neurosciences, 27(2), 72-74.

Rosenblatt, S. D., \& Crane, B. T. (2015). Influence of visual motion, suggestion, and illusory motion on self-motion perception in the horizontal plane. PLoS One, 10(11), e0142109.

Satterthwaite, F. E. (1946). An approximate distribution of estimates of variance components. Biometrics Bulletin, 2(6), 110-114.

Schwarz, G. (1978). Estimating the dimension of a model. The Annals of Statistics, 6(2), 461-464.

Schweig, S., Liebherr, M., Schramm, D., Brand, M., \& Maas, N. (2018). The impact of psychological and demographic parameters on simulator sickness. In SIMULTECH (pp. 91-97).

Seno, T., \& Sato, T. (2011). Vection can be induced without explicit motion signal using backscroll illusion. Japanese Psychological Research, 54(2), 218-222.

Seno, T., Sunaga, S., \& Ito, H. (2010). Vection aftereffects from expanding/contracting stimuli. Seeing and Perceiving, 23(4), 273 294.

Seno, T., Ito, H., \& Sunaga, S. (2011). Attentional load inhibits vection. Attention, Perception, \& Psychophysics, 73(5), 1467-1476.

Seno, T., Ito, H., \& Sunaga, S. (2012). Vection can be induced in the absence of explicit motion stimuli. Experimental Brain Research, 219(2), 235-244.

Seno, T., Kitaoka, A., \& Palmisano, S. (2013). Vection induced by illusory motion in a stationary image. Perception, 42(9), 1001-1005.

Seno, T., Sawai, K. I., Kanaya, H., Wakebe, T., Ogawa, M., Fujii, Y., \& Palmisano, S. (2017). The oscillating potential model of visually induced vection. i-Perception, 8(6), 2041669517742176.

Seya, Y., Tsuji, T., \& Shinoda, H. (2014). Effect of depth order on linear vection with optical flows. $i$-Perception, 5(7), 630-640.

Seya, Y., Shinoda, H., \& Nakaura, Y. (2015). Up-down asymmetry in vertical vection. Vision Research, 117, 16-24.

Shapiro, A. G., \& Todorovic, D. (Eds.). (2016).The Fraser-Wilcox illusion and its extension. In The Oxford compendium of visual illusions (pp. 500-511). Oxford University Press.
Shepherd, A. J. (2000). Visual contrast processing in migraine. Cephalalgia, 20(10), 865-880.

Shepherd, A. J., Hine, T. J., \& Beaumont, H. M. (2013). Color and spatial frequency are related to visual pattern sensitivity in migraine. Headache: The Journal of Head and Face Pain, 53(7), 1087-1103.

Smart Jr., L. J., Stoffregen, T. A., \& Bardy, B. G. (2002). Visually induced motion sickness predicted by postural instability. Human Factors, 44(3), 451-465.

Stanney, K. M., Hale, K. S., Nahmens, I., \& Kennedy, R. S. (2003). What to expect from immersive virtual environment exposure: influences of gender, body mass index, and past experience. Human Factors, 45(3), 504-520.

Team, R. C. (2019). R Foundation for Statistical Computing. R: A language and environment for statistical computing.

Telford, L., \& Frost, B. J. (1993). Factors affecting the onset and magnitude of linear vection. Perception \& Psychophysics, 53(6), 682-692.

Thornton, I. M., \& Zdravković, S. (2020). Searching for illusory motion. Attention, Perception, \& Psychophysics, 82(1), 44-62.

Tomimatsu, E., Ito, H., Sunaga, S., \& Remijn, G. B. (2011). Halt and recovery of illusory motion perception from peripherally viewed static images. Attention, Perception, \& Psychophysics, 73(6), $1823-1832$.

Turner, M., \& Griffin, M. J. (1999). Motion sickness in public road transport: the effect of driver, route and vehicle. Ergonomics, 42(12), 1646-1664.

Ujike, H., Ukai, K., \& Nihei, K. (2008). Survey on motion sickness-like symptoms provoked by viewing a video movie during junior high school class. Displays, 29(2), 81-89.

Vedamurthy, I., Nahum, M., Bavelier, D., \& Levi, D. M. (2015a). Mechanisms of recovery of visual function in adult amblyopia through a tailored action video game. Scientific Reports, 5, 8482.

Vedamurthy, I., Nahum, M., Huang, S. J., Zheng, F., Bayliss, J., Bavelier, D., \& Levi, D. M. (2015b). A dichoptic custom-made action video game as a treatment for adult amblyopia. Vision Research, 114, 173-187.

Webb, N. A., \& Griffin, M. J. (2003). Eye movement, vection, and motion sickness with foveal and peripheral vision. Aviation, Space, and Environmental Medicine, 74(6), 622-625.

Weech, S., Kenny, S., \& Barnett-Cowan, M. (2019). Presence and cybersickness in virtual reality are negatively related: a review. Frontiers in Psychology, 10, 158.

Winter, B. (2013). Linear models and linear mixed effects models in R: Tutorial 11. arXiv preprint arXiv:1308.5499.

Witmer, B. G., \& Singer, M. J. (1998). Measuring presence in virtual environments: a presence questionnaire. Presence, 7(3), 225-240.

Publisher's Note Springer Nature remains neutral with regard to jurisdictional claims in published maps and institutional affiliations. 